\title{
Design of Silicon MMI Crossing Laterally Coupled Microring Resonators
}

\author{
Hui Chen and Andrew W. Poon \\ Department of Electrical and Electronic Engineering, The Hong Kong University of Science and Technology, \\ Clear Water Bay, Hong Kong SAR, China \\ Tel: (852)-2358-7905; Fax: (852)-2358-1485; Email: eeawpoon@ust.hk
}

\begin{abstract}
We design a silicon MMI crossing laterally coupled microring resonator-based cross-connect filter. Numerical simulations suggest our design exhibits reduced insertion loss, reduced crosstalk, and more symmetric Lorentzian lineshapes as compared with the conventional plain-crossing-microring design.

(C) Optical Society of America

OCIS codes: (230.5750) Resonators; (230.3120) Integrated optics devices
\end{abstract}

High-density integration of microresonator arrays with two-dimensional waveguide cross-grids have long been proposed as a possible architecture for VLSI photonics [1]. However, conventional (plain) waveguide crossings often exhibit considerable insertion loss and crosstalk at the crossing junction, hence limits the number of crossing nodes. There exists various proposed schemes in the literature for improving the waveguide crossing characteristics, such as modifying the waveguide intersections by use of microresonators [2], elliptical tapering [3], and multimode interference (MMI) structures [4]. In this summary, we outline our initial design and analysis of using a MMI-based waveguide crossing laterally coupled with a microring resonator as a potential building block for microresonator arrays. We consider high-index-contrast silicon-based designs in order to achieve highly compact designs that are potentially wavelength tunable by means of silicon electro-optic effects.

Fig. 1(a) shows the schematic of a MMI crossing laterally coupled microring resonator. The MMI crossing consists of two orthogonal MMI sections with length $L_{m} \approx 2 n w_{m}{ }^{2} / \lambda$, where $w_{m}$ is the MMI section width, $n$ is the effective refractive index of MMI waveguide fundamental mode, and $\lambda$ is the free space wavelength. Each MMI section generates a single self-image at the MMI center and output. The intersection region of the two MMI structures is then effectively a slab region. Light beam first focuses to form a self-image and then diverges to another half of the MMI. In contrast, light beam typically diverges in conventional waveguide crossing intersections. We connect the bus waveguide to the MMI section with a taper in order to spatially expand the waveguide mode field. This taper serves three essential purposes: (i) to reduce the transition loss between the bus waveguide and the MMI section, (ii) to reduce the divergence of the imaged beam at the crossing center, and (iii) to enhance the fabrication tolerance of the crossing

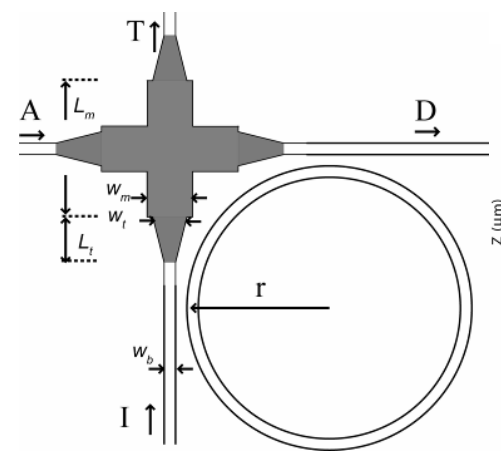

(a)

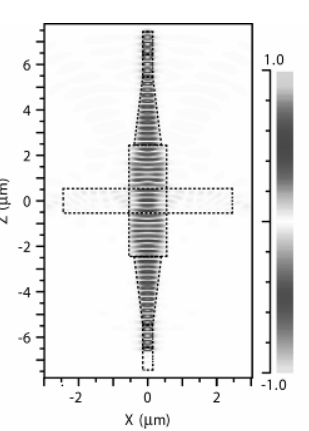

(b)

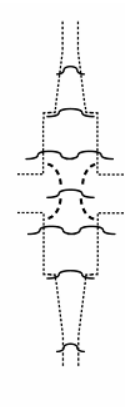

)

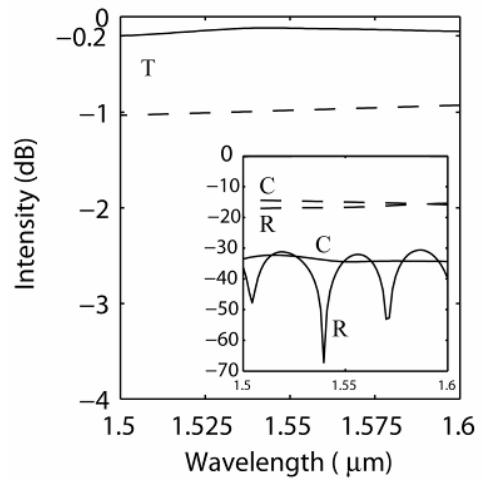

(c)

Fig 1. (a) Schematic of MMI crossing coupled microring resonator. MMI crossing (in gray) dimensions are: $w_{m}=$ $1.1 \mu \mathrm{m}, L_{m}=4.9 \mu \mathrm{m}, w_{t}=0.8 \mu \mathrm{m}, L_{t}=3 \mu \mathrm{m}, w_{b}=0.3 \mu \mathrm{m}$, and $r=8 \mu \mathrm{m}$. I: input, T: throughput, D: drop, A: add. (b) FDTD-simulated $\mathbf{H}$-field pattern (TE polarization) of the MMI crossing. Schematic shows the self-imaging and focusing in the MMI section. (c) Simulated throughput spectra of the MMI crossing (solid line) and plain crossing (dashed line) of $w_{b}=0.3 \mu \mathrm{m}$. Inset shows the simulated cross-port spectra and back-reflection spectra of the MMI crossing (solid line) and the plain crossing (dashed line). 


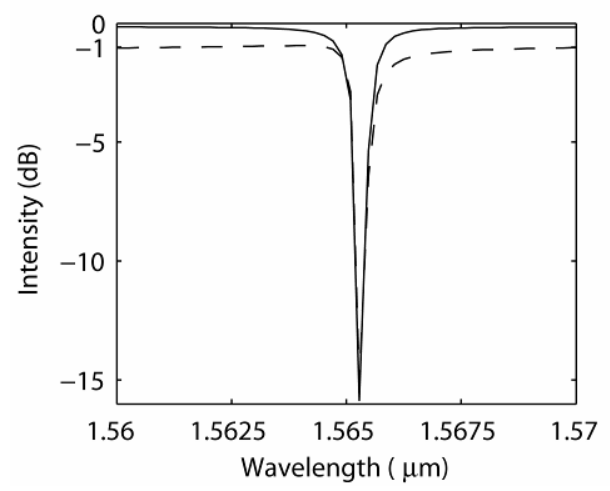

(a)

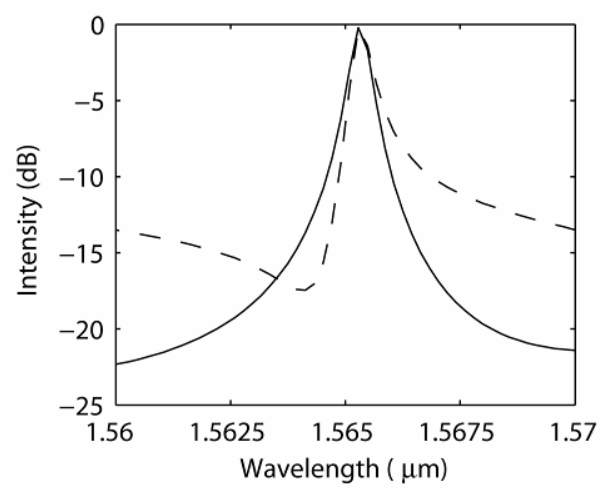

(b)

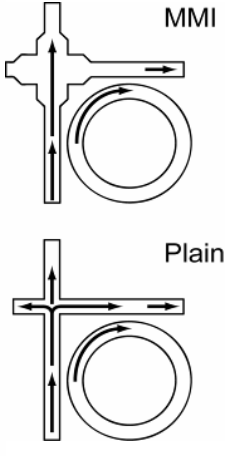

(c)

Fig. 2 Simulated spectra of MMI crossing (solid line) and plain crossing (dashed line) coupled microring resonator filters. (a) throughput-port spectra. (b) drop-port spectra. (c) Schematics of microring-coupled resonance channel in MMI-crossing design, and channel interference between the resonance channel and the crossing junction scattering channel in the plain-crossing design.

structure.

We design the MMI crossing for silica-clad silicon waveguide with bus waveguide width $w_{b}=0.3 \mu \mathrm{m}$. We first choose $w_{m}=1.1 \mu \mathrm{m}$ in order to support five waveguide modes for better self-image quality. Then we calculate $L_{m}$ in order to obtain two self-imaging positions. By using two-dimensional finite-difference time-domain (FDTD) method, we optimize the input mode width (to $0.8 \mu \mathrm{m}$ ) and $L_{m}$ (to $4.9 \mu \mathrm{m}$ ) in the MMI crossing configuration to maximize transmission. We choose the tapering width $\left(w_{t}\right)$ as the optimized input mode width and the tapering length $\left(L_{t}\right)$ equals to $3 \mu \mathrm{m}$ without introducing excessive radiation loss in the transition between the broadened mode and the output bus waveguide mode. Including the tapering structures, our optimized MMI crossing only occupies a small footprint of $\sim 11 \mu \mathrm{m} \times 11 \mu \mathrm{m}$. Fig. 1(b) shows the simulated TE-polarization field pattern distribution of the optimized MMI crossing. The field pattern confirms the self-imaging and focusing at the crossed MMI intersection, and suggests very low crosstalk. Fig. 1(c) shows the FDTD-simulated spectra of the MMI crossing in comparison with those of the plain crossing of width $w_{b}$. Our MMI crossing displays only $\sim-0.18 \mathrm{~dB}$ insertion loss, whereas the plain crossing exhibits $\sim-1 \mathrm{~dB}$ insertion loss. Moreover, the MMI crossing also serves to suppress the crosstalk from $\sim-15 \mathrm{~dB}$ for the plain crossing to less than $-30 \mathrm{~dB}$, and suppress the back-reflection from $\sim-17 \mathrm{~dB}$ for the plain crossing to below $-30 \mathrm{~dB}$.

We employ FDTD simulations to numerically study the proposed MMI crossing coupled microring resonator, and the plain crossing coupled microring resonator in silica-clad silicon-on-insulator substrates. In each case, we adopt an 8- $\mu$ m-radius microring resonator with a waveguide width of $0.3 \mu \mathrm{m}$. Fig. 2(a) shows the simulated throughput-port spectra for the MMI crossing design and the plain crossing design. Similar to the crossing-only simulations (Fig. 1(c)), the MMI-microring crossing design improves the insertion loss by $\sim 0.8 \mathrm{~dB}$. Fig. 2(b) shows the simulated drop-port spectra. The MMI-microring crossing demonstrates a more symmetric (Lorentzian) resonance lineshape, whereas the plain crossing design reveals a highly asymmetric resonance lineshape. We attribute the asymmetric resonance lineshape to Fano resonance interference effect between the Lorentzian resonance channel (through the microring) and the coherent background crosstalk channel (through crossing junction induced scattering), as shown in Fig. 2(c). In our MMI-crossing design, the undesirable junction-induced crosstalk is minimized, and thus the dropport spectrum only displays the Lorentzian resonance channel. Further device optimization and fabrication of the proposed MMI-microring crossing designs on thin silicon-on-insulator substrates are in progress.

[1] Y. Kokubun, Y. Hatakeyama, M. Ogata, S. Suzuki, and N. Zaizen, "Fabrication Technologies for Vertically Coupled Microring Resonator With Multilevel Crossing Busline and Ultracompact-Ring Radius,” IEEE J. of Sel. Top. in Quant. Electron., 11, 4 - 10 (2005)

[2] C. Manolatou, S. G. Johnson, S. Fan, P. R. Villeneuve, H. A. Haus, and J. D. Joannopoulos, “High-density integrated optics,” IEEE J. Ligthwave Technol., 17, 1682 - 1692 (1999)

[3] T. Fukazawa, T. Hirano, F. Ohno, and T. Baba, “Low loss intersection of Si photonic wire waveguides,” Jpn. J. Appl. Phys., 43, 647-646 (2004)

[4] H. Liu, H. Tam, P. K. A. Wai, and E. Pun, “Low-loss waveguide crossing using a multimode interference structure,” Opt. Comm., 241, 99-104 (2004) 